Case report

\title{
Intramural hematoma of the esophagus after thrombolysis for ischemic stroke ${ }^{\hbar t}$
}

\section{Abstract}

Intramural dissecting hematoma is an unusual esophageal condition with a threatening presentation but excellent prognosis when managed conservatively. We report the case of an 88-year-old woman who developed an intramural hematoma of the esophagus after intravenous thrombolysis for an acute ischemic stroke. Before thrombolysis, nasogastric intubation was attempted unsuccessfully. She was kept on nil by mouth, intravenous hydration, proton pump inhibitor, antiemetics, and an antibiotic initiated 2 days before for periodontal disease. The esophageal hematoma regressed, and she resumed oral diet asymptomatically. To our knowledge, this is the first report of this type of lesion after thrombolysis for an ischemic stroke. A brief discussion and literature review are presented.

An 88-year-old woman presented to the emergency department with right conjugate gaze deviation, left central facial palsy, and left hemiparesis (National Institutes of Health Stroke Scale (NIHSS), 11). She had a prior history of arterial hypertension and dyslipidemia and was antiaggregated with acetylsalicylic acid. Two days before, she had initiated amoxicillin for periodontal disease. Brain computed tomographic (CT) scan showed no acute vascular lesions. An ischemic stroke was presumed, nasogastric intubation was attempted unsuccessfully, and thrombolysis with intravenous alteplase $(0.9 \mathrm{mg} / \mathrm{kg})$ was administered 120 minutes after symptoms onset. She was admitted to the stroke unit and gradually recovered from her neurologic deficits persisting only mild facial asymmetry (NIHSS, 1 ). Reevaluation brain CT scan showed an acute ischemic lesion in the cortical territory of right median cerebral artery and a minor left parietal hematoma. Further investigation revealed atrial fibrillation, and a cardioembolic stroke was assumed.

On the third day after admission, she presented retrosternal discomfort on swallowing and a single episode of blood-streak food vomit. She remained hemodynamically stable, but hemoglobin level dropped from 11.7 to $8.1 \mathrm{~g} / \mathrm{dL}$. Upper endoscopy showed a small clot immediately below the cricopharyngeus and, distally, a wide elevation of purplish esophageal wall causing a marked narrowing of the esophageal lumen. Thoracic CT scan confirmed a tubular thickening of the esophagus with a longitudinal extension of approximately $20 \mathrm{~cm}$, suggesting an extensive intramural hematoma (Figure). Gastrografin swallow showed no leaks.

Amoxicillin was maintained for 7 days, and the patient was kept on nil by mouth, intravenous hydration, proton pump inhibitor, and antiemetics. Ten days after, CT scan showed partial resolution of the esophageal thickening with no oral contrast leaks.

Anticoagulation with enoxaparin was initiated 7 days after detection of the hematoma, without complications. Oral intake was resumed with

\footnotetext{
Conflicts of interest: All authors declare no conflicts of interest.
}

liquids on the second day after diagnosis and progressed gradually to her regular diet.

This case describes an esophageal dissecting intramural hematoma after trauma during attempt for nasogastric intubation and facilitated by thrombolysis.

Esophageal intramural hematoma is a rare and underrecognized condition. It is more common in women, and its incidence increases with age [1]. Almost $80 \%$ of patients present at least 2 of the 3 typical features: chest pain, hematemesis, and dysphagia/odynophagia [2]. Recognition of esophageal hematoma is important, as it may mimic other diseases [3] (eg, myocardial infarction or pulmonary embolism), which may require treatments that are contraindicated in the presence of a hematoma.

Computed tomographic scan with both oral and intravenous contrasts is the preferred investigation method. It can identify the hematoma, assess associated complications, and exclude other differential diagnoses $[4,5]$. Upper endoscopy usually reveals a large fluctuant purplish hematoma compressing the lumen with pale mucosa over its surface [5].

Once diagnosis has been established, conservative treatment with intravenous fluids, antiemetics, and proton pump inhibitors is recommended, with resolution of symptoms occurring within 7 to 14 days without sequelae in most cases $[4,5]$.

There are few reports of intramural esophageal hematomas after thrombolysis for myocardial infarction [6] or pulmonary embolism [7], after placement of nasogastric [8] or orotracheal [1] tubes. To our knowledge, this is the first report of this type of injury after thrombolysis for an acute ischemic stroke, a situation where trauma from nasogastric tube placement is common. Awareness for this condition is, therefore, vital.

A written authorization was obtained from the patient.

Mario Jorge Silva, MD* Gastroenterology Department, Central Lisbon Hospital Centre Alameda de Santo António dos Capuchos, 1169-050, Lisboa, Portugal NOVA Medical School, Campo dos Mártires da Pátria 130, 1169-056 Lisboa, Portugal

${ }^{*}$ Corresponding author. Centro Hospital de Lisboa Central EPE-Serviço de Gastrenterologia, Alameda de Santo António dos Capuchos, 1169-050 Lisboa.

Tel.: + 351213136320 .

E-mail address: mariojorgesilva01@gmail.com

Joana Saiote, MD

Gastroenterology Department, Central Lisbon Hospital Centre Alameda de Santo António dos Capuchos, 1169-050, Lisboa, Portugal NOVA Medical School, Campo dos Mártires da Pátria, 130, 1169-056 Lisboa, Portugal 


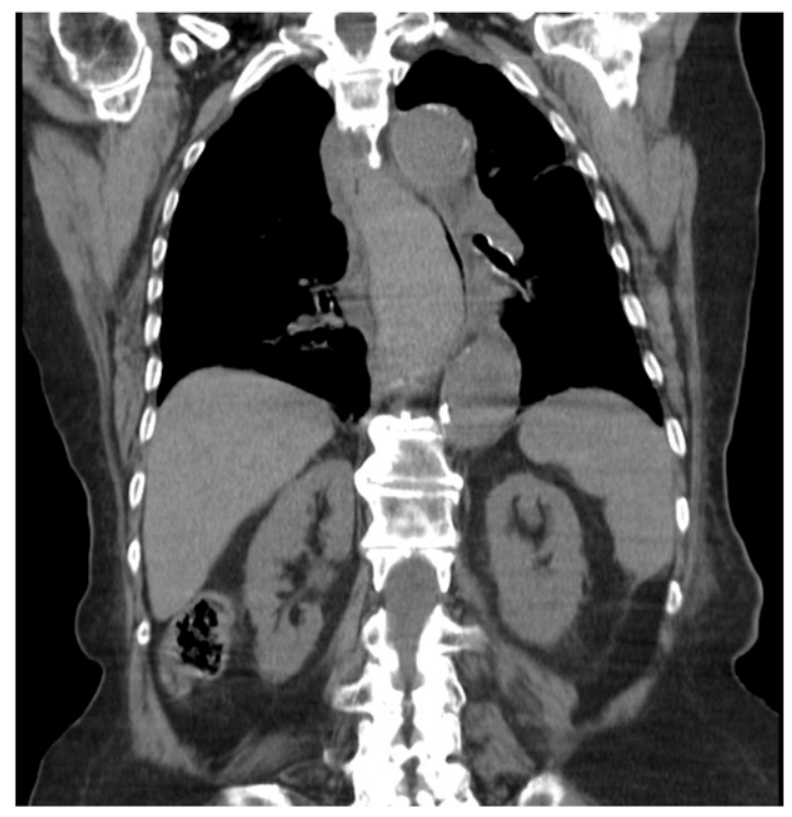

Figure. Extensive esophageal intramural hematoma on CT scan.

Vera Salvado, MD

Internal Medicine Department 1.2, Central Lisbon Hospital Centre, Rua José António Serrano, 1150-199, Lisboa, Portugal
Ana Paiva Nunes, MD

Stroke Unit, Central Lisbon Hospital Centre, Rua José António Serrano

1150-199, Lisboa, Portugal

Pedro Duarte, MD

Gastroenterology Department, Central Lisbon Hospital Centre, Alameda de Santo António dos Capuchos, 1169-050, Lisboa, Portugal

http://dx.doi.org/10.1016/j.ajem.2014.08.069

\section{References}

[1] Wang AY, Riordan RD, Yang N, Hiew CY. Intramural haematoma of the oesophagus presenting as an unusual complication of endotracheal intubation. Australas Radio 2007;51(Suppl):B260-4.

[2] Cullen SN, McIntyre AS. Dissecting intramural haematoma of the oesophagus. Eur J Gastroenterol Hepatol 2000;12(10):1151-62.

[3] Tay YK, Tay JY, Dandie L, Gribbin J. Intramural hematoma of the esophagus mimicking hemorrhaging esophageal cancer. Ann Gastroenterol 2013;26(1):74-6.

[4] Beumer JD, Devitt PG, Thompson SK. Intramural oesophageal dissection. ANZ J Surg 2010;80(1-2):91-5.

[5] Modi P, Edwards A, Fox B, Rahamim J. Dissecting intramural haematoma of the oesophagus. Eur J Cardiothorac Surg 2005;27(1):171-3.

[6] Huddy J, Kotecha A, Dussek J, McNair A. Dissecting intramural hematoma of the esophagus after thrombolysis for myocardial infarction. Gastrointest Endosc 2005 61(2):340-3.

[7] Hong M, Warum D, Karamanian A. Spontaneous intramural esophageal hematoma secondary to anticoagulation and/or thrombolysis therapy in the setting of a pulmonary embolism: a case report. J Radiol Case Rep 2013:7(2):1-10.

[8] Hsu CC, Changchien CS. Endoscopic and radiological features of intramural esophageal dissection. Endoscopy 2001;33(4):379-81. 